\title{
CARBAMAZEPINE INDUCED TOXIC EPIDERMAL NECROLYSIS: A CASE REPORT
}

\author{
SHERIN ALEXANDER ${ }^{1}$, DEVAYANI SANTHU ${ }^{1}$, NARENDRAN T T리 JULIE MARIAM JOSHUA ${ }^{1}$, MEENU VIJAYAN ${ }^{2 *}$, \\ JACOB THOMAS
}

${ }^{1}$ Department of Pharmacy Practice, Amrita School of Pharmacy, Amrita Vishwa Vidyapeetham, AIMS Health Sciences Campus, Kochi, Kerala, India. ${ }^{2}$ Department of Pharmacy Practice, Amrita School of Pharmacy, Amrita Vishwa Vidyapeetham, AIMS Health Sciences Campus, Kochi, Kerala, India. ${ }^{3}$ Department of Dermatology, Amrita Institute of Medical Sciences, Amrita Vishwa Vidyapeetham, AIMS Health Sciences Campus, Kochi, Kerala, India. Email: meenuvijayan@aims.amrita.edu

Received: 04 July 2018, Revised and Accepted: 11 September 2018

ABSTRACT

Toxic epidermal necrolysis (TEN) is a life-threatening dermatological disorder characterized by erythematous lesions, necrosis, exfoliation, sepsis, and death. This may be due to different reasons such as chemical exposure, systemic infections, or some reactions of drugs such as carbamazepine, phenytoin, allopurinol, lamotrigine, and nonsteroidal anti-inflammatory drugs. In this case, the patient was on carbamazepine for the management of alcohol withdrawal symptoms. Moreover, this might be the reason for the occurrence of TEN. For his condition, he had given corticosteroids, antibiotics for sepsis, and other supportive measures.

Keywords: Toxic epidermal necrolysis, Carbamazepine, Erythematous lesion.

(c) 2018 The Authors. Published by Innovare Academic Sciences Pvt Ltd. This is an open access article under the CC BY license (http://creativecommons. org/licenses/by/4. 0/) DOI: http://dx.doi.org/10.22159/ajpcr.2018.v11i12.28294

\section{INTRODUCTION}

The skin is the external covering for humans and helps us interact with our surroundings. The skin is, however, prone to a number of ailments, either due to the substances they come in contact with or due to the substances we consume. Toxic epidermal necrolysis (TEN) such as Stevens-Johnson syndrome (SJS) is rare reactions to the drug that is seriously cutaneous and could even lead to death $[1,2]$. In up to $95 \%$ of its occurrences, drugs are usually the major cause [3,4]. Quinolones, penicillins, and macrolides such as erythromycin, chloramphenicol, and sulfonamides are antibacterial drugs that are associated with the skin condition. TEN is also believed to be triggered by anticonvulsants such as carbamazepine, a derivative of antiepileptic iminostilbene that is used for treating epilepsy. Carbamazepine aids the reduction of nerve impulses in the body that causes pain and seizures, thereby making it effective for treating epilepsy and pain-related ailments. It is also effective for the treatment of bipolar disorder and for the management of alcohol withdrawal symptoms. The most frequently observed adverse effects of carbamazepine include hematological disorders, vomiting, and nausea. Chronic therapy with antiepileptic drugs such as carbamazepine possesses a significant risk for developing osteoporosis and anemia [5]. There are rare cases, where it leads to reactions such as TEN and SJS, that is, life-threatening. Carbamazepine-induced systemic lupus erythematosus is another rare and serious adverse drug reaction that has been reported [6].

Some common indications of TEN include bullous detachment of the mucous membranes and epidermis, necrosis, and erythema, resulting in exfoliation and ultimately leading to sepsis or death. TEN is a condition that is normally unique to every individual, thereby, making it difficult to predict its occurrence. This has also made it difficult to properly explain the "TEN" pathophysiology. A number of theories have been widely accepted. Recent studies have revealed a strong association between HLA-B*1502 and CBZ-induced TEN/SJS [7]. TEN is believed to be an immune reaction that is cytotoxic, fighting keratinocytes that display antigens that are foreign to the body. It is similar to the hypersensitivity reaction, recognized by an antigen initial exposure reaction that is delayed, and a quick increasing reaction is noticed when the exposure is repeated.

The careful and prompt recognition of the problem and removal of all potential agents causing it is the best way to manage TEN. Supportive care pending the regeneration of the epithelium is the most effective treatment for TEN. The major supportive measure includes protective dressings, pain management, nutritional support, electrolyte, and fluid balance as well as isolation. Patients transfer to intensive or burn care unit early enough is a great way of considerably reducing the length of hospitalization, mortality rate, and infection rate. The agent leading to the reaction must be stopped instantly. Titrations and fluids administration, in line with urine output and central venous pressure, are vital. On an average, 3-4 $\mathrm{L}$ of fluid are required for those individuals who have $50 \%$ of the surface area of their body affected. Pharmacotherapy in TEN is carried out with the aim of avoiding complications and reducing morbidity. While agents including antiseptics, analgesics, anticoagulants, antihistamines, antibiotics, and crystalloids agents are considered vital for supportive care, there is no specific modality for treatment. TEN management with corticosteroids is still controversial.

\section{CASE REPORT}

A male patient, aged 40 years, was brought to our hospital with complaints of bullous lesions followed by skin peeling and erosions. About 2 weeks back, the patient had been to a deaddiction center and was started on carbamazepine (single dose of $200 \mathrm{mg}$ ), baclofen, and thiamine $150 \mathrm{mg}$. Later, he had developed bullous lesions (initially started over lips, then eyelids, trunk, and limbs) and low-grade fever and was given intravenous (IV) steroids.

On admission, patients were conscious but had mild disorientation and vitals were stable. Physical examination revealed multiple erythematous and bullous lesions all over the body with erosions in the oral cavity and eyelids. Systemic examination was within the normal limits. Inflammatory markers were elevated (C-reactive protein $-1773.33 \mathrm{nmol} / \mathrm{L}$ and erythrocyte sedimentation rate $-23 \mathrm{~mm} / \mathrm{h}$ ) with deranged liver function test with mildly raised total bilirubin (25.99 umol/L) and direct bilirubin ( $8.55 \mathrm{umol} / \mathrm{L})$ and dyselectrolytemia (calcium - $1.9 \mathrm{mmol} / \mathrm{L}$, phosphorous - $0.68 \mathrm{mmol} / \mathrm{L}$, sodium - $124 \mathrm{mmol} / \mathrm{L}$, and potassium - $3.4 \mathrm{mmol} / \mathrm{L}$ ). Urine routine done was found to be normal.

The patient was initially admitted under dermatology department and he was started on injection hydrocortisone $100 \mathrm{mg}$, and IV fluid with a diagnosis of TEN due to carbamazepine. The patient was nursed in reverse isolation in view of extensive skin involvement and was 
started empirically on tablet azithromycin $500 \mathrm{mg}$ optometrist (OD). Ophthalmology consultation was sought for erosions over upper and lower eyelids and advised refresh liquigel, Genteal gel, and Milflox gel (ciprofloxacin $0.3 \%$ and dexamethasone $0.1 \%$ ). Psychiatry consultation was sought for further treatment of alcohol withdrawal. Urine culture showed growth of Pseudomonas aeruginosa. Physical examination revealed that the patient was tachycardic, febrile and on view of his raised procalcitonin levels $(19.85 \mathrm{ug} / \mathrm{L})$, he was started on injection Piptaz (piperacillin/tazobactam) 3.375 g Q8H, injection teicoplanin $400 \mathrm{mg} \mathrm{Q24H}$, and injection thiamine $100 \mathrm{mg}$ OD. He was continued on injection hydrocortisone $100 \mathrm{mg}$ TID and all eye drops refresh liquigel Q6H, Genteal gel, and Milflox eye drops TID. Dyselectrolytemia was corrected and saline compresses were given over the lesions.

Once the patient becomes hemodynamically stable, he was started on tablet cyclosporine $100 \mathrm{mg}$ OD. Skin lesions over the face and trunk started forming crusts and were gradually healing. Fusidic acid was added for local application over the erosions. Lesions started healing and reepithelization was seen. IV steroids (injection hydrocortisone) were tapered to twice daily and then once a day. Injection Piptaz and teicoplanin were continued for 14 days. The patient was hemodynamically stable at the time of discharge. Hence, injection hydrocortisone $(100 \mathrm{mg})$ was changed to tablet Wysolone (prednisolone) $30 \mathrm{mg}$ and he was advised to review in psychology outpatient department for counseling.

\section{DISCUSSION}

Our patient is a 40-year-old male with a history of chronic alcoholism. He was started on carbamazepine for the management of alcohol withdrawal symptoms. Moreover, this might have contributed to the occurrence of TEN. Naranjo adverse drug reaction probability scale was used to score and assess the causality of TEN in this context, and it was found to be 8 (probable). The patient recovered after stopping the drug. Challenge was not carried out due to the potential risk involved.

In our case, the patient was treated with injection hydrocortisone and when he was hemodynamically stable, it was switched to oral form. He was septic at the time of admission, but he improved on IV antibiotics without any complications or sequelae.

The use of IV corticosteroids in the management of TEN has limited trials and is still controversial. Hypothetically, corticosteroids increase the chance of sepsis and protein catabolism and decrease the rate of reepithelialization, their use is contraindicated in patients with extensive skin detachment.

However, in a retrospective study of 281 patients with SJS/TEN, only $18 \%$ mortality rate was observed in the corticosteroid group, while in the IV immunoglobulin and supportive care group, it was $25 \%$. The odds ratio of death for those patients who were on steroids compared to those on supportive cares alone was 0.6 , demonstrating a potential benefit $[8,9]$.

\section{CONCLUSION}

TEN is an uncommon but fatal skin disorder. Hence, early detection and diagnosis (through biopsy) are very important. The prompt identification and withdrawal of the offending drug are crucial. In our study, the culprit drug was carbamazepine, which was prescribed for the management of alcohol withdrawal symptoms. In such cases, Levetiracetam can be the drug of choice $[10,11]$. The United States Food and Drug Administration has recommended for prospective screening for specific genotypes contributing to its occurrence like HLA-B*1502 allele, before the commencement of treatment with suspected drugs [7]. This could possibly decrease the burden of incidence of TEN. Future prospectives should be aimed at revamping the prevention and management of occurrence of such devastating form of adverse drug reactions.

Even though the use of IV steroids is controversial, it did not cause any significant variance in outcome in our case. Along with the medication therapy, supportive care is also an essential factor for the mitigation of this condition.

\section{ACKNOWLEDGMENT}

We would like to thank our department of pharmacy practice for their valuable suggestions in bringing the case report to completion. We would also like to thank the hospital authority, patient, and his bystanders for their cooperation.

\section{AUTHORS' CONTRIBUTIONS}

Identification and conceptualization of work: Sherin Alexander and Devayani Santhu, Wrote the manuscript, checked the references: Sherin Alexander, Devayani Santhu, Narendran T, and Julie Mariam Joshua. Compiled the literature sources, interpreted data: Julie Mariam Joshua. Helped in data interpretation and reference checking: Dr. Jacob Thomas. Wrote parts of the manuscript, acted as corresponding author, corrections and suggestions regarding manuscript: Meenu Vijayan.

\section{CONFLICTS OF INTEREST}

There are no conflicts of interest.

\section{REFERENCES}

1. Das AR, Saikeerthana PC, Raj K, Anila KN. Carbamazepine induced toxic epidermal necrolysis-a rare case report. Int J Pharm Sci Rev Res 2016:40:18-9.

2. Lassen A, Piepgras D, Chyatte D, Rizzolo D. Trigeminal neuralgia: Diagnosis and medical and surgical management. J Am Acad Physician Assistants 2011;24:20-5

3. McNamara JO. Pharmacotherapy of the epileptics. In: Hardman JG, Limbird LE, Gilman AG, editors. Goodman and Gilman's the Pharmacological Basis of Therapeutics. New York: McGraw Hill Medical; 2011. p. 594-5.

4. Wu Y, Sanderson JP, Farrell J, Drummond NS, Hanson A, Bowkett E. Activation of T cells by carbamazepine and carbamazepine metabolites. J Allergy Clin Immunol 2006;118:233-41.

5. Angel V, Philip B, Krishnakumar M, Kuriakose F, Rodrigues PA. Assessment of osteoporosis and anaemia risk in patients on anticonvulsant therapy. Int J Pharm Pharm Sci 2016;8:404-8.

6. Keerthana PC, Anila KN. Carbamazepine-induced SLE-a rare and serious ADR. Int J Pharm Pharm Sci 2017;9:319-20.

7. Ferrell PB, McLeod H. Carbamazepine, HLA-B*1502 and risk of Stevens-Johnson syndrome and toxic epidermal necrolysis: US FDA recommendations. Pharmacogenomics J 2008;9: 1543-6.

8. Alexander S, Anila KN. A case report on anti-N-methyl-D-aspartate receptor encephalitis. Asian J Pharm Clin Res 2017;10:3-4.

9. Patil SB, Roy AG, Vinayan KP. Clinical profile and treatment outcome of febrile infection-related epilepsy syndrome in South Indian children. Ann Indian Acad Neurol 2016;19:188.

10. Muller CA, Schafer M, Schneider S, Heimann HM, Hinzpeter A, Volkmar K, et al. Efficacy and safety of levetiracetam for outpatient alcohol detoxification. Pharmacopsychiatry J 2010;43:184-9.

11. Sudan TS, Vinayan KP, Roy AG. Clinical, MRI and electrographic characteristics of three children with hemiconvulsion-hemiplegia/ hemiconvulsion-hemiplegia-epilepsy (HH/HHE) syndrome - A rare childhood epileptic encephalopathy. Int J Epilepsy 2017;4:79-86. 